\title{
Gilbert Ouy, Christine Reno, Inès Villela-Petit, Album Christine de Pizan
}

\section{Maria Colombo Timelli}

\section{(2) OpenEdition}

1 Journals

\section{Édition électronique}

URL : http://journals.openedition.org/studifrancesi/2087

DOI : 10.4000/studifrancesi.2087

ISSN : 2421-5856

Éditeur

Rosenberg \& Sellier

\section{Édition imprimée}

Date de publication : 1 avril 2014

Pagination : 122-123

ISSN : 0039-2944

\section{Référence électronique}

Maria Colombo Timelli, « Gilbert Ouy, Christine Reno, Inès Villela-Petit, Album Christine de Pizan », Studi Francesi [En ligne], 172 (LVIII | I) | 2014, mis en ligne le 01 avril 2014, consulté le 18 septembre 2020 URL : http://journals.openedition.org/studifrancesi/2087 ; DOI : https://doi.org/10.4000/studifrancesi. 2087

Ce document a été généré automatiquement le 18 septembre 2020.

\section{(c)}

Studi Francesi è distribuita con Licenza Creative Commons Attribuzione - Non commerciale - Non opere derivate 4.0 Internazionale. 


\title{
Gilbert Ouy, Christine Reno, Inès Villela-Petit, Album Christine de Pizan
}

\author{
Maria Colombo Timelli
}

\section{RÉFÉRENCE}

GILBERT OUY, CHRISTINE RENO, INÈS VILLELA-PETIT (OLIVIER DELSAUX \& TANIA VAN HEMELRYCK

éditeurs et collaborateurs, Avec les conseils de James Laidlow \& Marie-Thérèse

Gousset), Album Christine de Pizan, Turnhout, Brepols, 2012, pp. 800.

1 Réalisé par deux des plus grands spécialistes de Christine de Pizan - Christine Reno et le regretté Gilbert Ouy - avec Inès Villela-Petit, conservatrice à la BnF et histoirenne de la miniature, sans négliger la collaboration d'une équipe de recherche plus large, ce répertoire réunit 54 notices consacrées à la description codicologique approfondie des manuscrits originaux recensés à ce jour. L'inventaire est précédé d'une longue préface en trois volets.

2 L'Introduction générale (pp. 15-38) présente rapidement l'histoire et les raisons d'être d'un ouvrage fondé sur les compétences de Christine, responsable autant du contenu de ses œuvres que de leur copie et décoration. Dans ce cadre, il est intéressant de rappeler quelques acquis relativement récents: l'existence des trois «mains» qui se sont partagé le travail de transcription ( $P, R, X$, chacune utilisant aussi une écriture cursive plus hâtive désignée par $\left.P^{\prime}, R^{\prime}, X^{\prime}\right)$, leurs caractéristiques respectives et les tâches qu'elles ont assumées; si X est désormais reconnue comme la main de Christine elle-même, et si on a pu attribuer une identité à $\mathrm{R}$ (un certain «P. de La Croix»), $\mathrm{P}$ demeure toujours anonyme. Des pages à part (39-88) sont consacrées aux «enlumineurs ornemanistes», à savoir les artistes qui se chargeaient de la décoration des manuscrits en dehors des miniatures proprement dites: lettrines filigranées, lettrines et bordures à vigneture, lettres champies. Là encore, les informations fournies sont riches et les auteurs n'hésitent pas à rappeler, à côté d'éléments bien connus de tout médiéviste ayant à peine feuilleté un manuscrit (par exemple, la hiérarchisation des lettrines dans 
l'organisation d'un texte), des notions beaucoup plus techniques, qui permettent de mieux apprécier, au-delà de l'originalité des manuscrits de Christine, les habitudes d'ornementation dans les manuscrits de l'époque. Une douzaine de mains de ces enlumineurs ont pu être reconnues, dont les caractéristiques principales sont présentées dans des paragraphes spécifiques: les filigraneurs «aux lys losangés», «aux couleurs insolites», «aux lys courbes» pour le premier groupe; et, pour les vignetures: l'atelier «aux échancrures», les ornemanistes «aux araignées», «aux brins de fantaisie», Pierre Gibert, l'«assistant aux trois couleurs», les ornemanistes "aux trèfles», «aux radicelles», «à la résille». Les peintres enlumineurs sont présentés dans la troisième partie (pp. 91-170). Non seulement l'œuvre de Christine de Pizan «compte parmi les plus richement illustrées de tous les auteurs du Moyen Âge», mais l'attention qu'elle consacrait au rapport texte-image(s), imposait qu'un tel sujet retînt l'attention. Après avoir examiné le cas emblématique des enluminures centrées sur la Salle du château de Fortune dans la Mutation, dont est fort heureusement conservée l'inscription, l'attention se focalise sur les onze peintres auxquels Christine a confié l'illustration de ses manuscrits entre 1400 et 1414; cinq dominent surtout: le Maître de la Pastoure, le Maître du Couronnement de la Vierge, avec le Maître de l'Épître Othéa, le Maître de la Cité des dames et le Maître d'Egerton. Les paragraphes consacrés à chacun d'entre eux permettent de mettre en relief l'importance de leur production et leurs traits saillants.

Un nombre considérable de reproductions - malheureusement toutes en noir et blancaccompagne ces pages, et permet même au lecteur peu ou pas spécialiste de reconnaître assez vite les systèmes graphiques, les différents types de décoration, ou encore les techniques d'illustration objet de l'analyse.

Le Répertoire sépare les manuscrits-recueils, traités à part, des manuscrits monotextuels, ceux-ci divisés entre manuscrits en parchemin et en papier. Comme on l'a dit, il s'agit de 54 notices détaillées, comprenant les entrées suivantes: cote, titre, contenu, histoire du ms (date de confection, possesseurs), description matérielle (matériaux: support, encres; préparation: piqûres, réglure; mise en page; organisation: signatures, réclames, foliotation; travail d'écriture: texte, corrections, rubriques; décoration; illustration; reliure); commentaire; bibliographie. Si les aspects purement codicologiques semblent primer, de fait l'examen des programmes iconographiques, ainsi que la section "commentaire» offrent des remarques du plus haut intérêt sur l'interprétation des textes concernés.

5 Pour ce qui est des manuscrits-recueils (notices aux pp. 186-343), cinq copies sont en cause: les deux volumes de Chantilly, Musée Condé, 492 et 493, qui conservent le recueil le plus ancien des œuvres de Christine, le ms. fr. 12779 de la BnF, les «manuscrits du Duc», vraisemblablement Louis d'Orléans (BnF, fr. 835, fr. 606, fr. 836, fr. 605, fr. 607), le célèbre «manuscrit de la Reine» (London, BL, Harley 4431, en deux volumes); à ceux-ci il faut encore ajouter le ms BnF, fr. 603, qui transmet Le livre de faiz d'armes et de chevallerie et la Mutacion de Fortune, et deux fragments qui attestent l'existence de deux autres manuscrits du début du XVe siècle. L'Introduction (pp. 173-185) porte surtout sur le contenu des recueils conservés, l'ordre de présentation des œuvres, et les différences souvent introduites en fonction $\mathrm{du} / \mathrm{de}$ la destinataire: la lecture de ces pages est éclairante pour le lecteur non spécialiste, car la synthèse des informations exposées en détail dans la notice de chaque manuscrit permet de les encadrer dans une vision plus vaste. 
6 Suivent les manuscrits monotextuels en parchemin: Epistre Othea (BnF, fr. 848), Debat de deux amans (BnF, fr. 1740 et KBR, 11034), Dit de la pastoure (BnF, fr. 2184), Chemin de lonc estude (KBR, 10983 et 10982; BnF, fr. 1188 et fr. 1643), Mutacion de Fortune (cinq mss, dont un en mains privées), Fais et bonnes meurs de Charles $V$ (quatre mss), Cité des dames (cinq mss), Epistre de la reine (BnF, fr. 580); puis les manuscrits sur papier: Advision Christine (BnF, fr. 1176; KBR, 10309; la description d'un troisième, en mains privées, est partielle, le ms en question ayant été examiné il y a longtemps: voir note 6 p. 606), Trois Vertus (Boston, PL, fr. Med. 101; BnF, n.a.fr. 25636), Prodommie de l'omme (Vatican, BAV, Reg. lat. 1238), Corps de policie (quatre mss), Sept psaumes allegorisés (KBR, 10987; BnF, n.a.fr. 4792; les informations sur un troisième $\mathrm{ms}$, dans une collection privée, sont de seconde main), Faits d'armes et de chevalerie et Livre de paix (KBR, 10476 et 10366), Epistre de la prison de vie humaine (BnF, fr. 24786: il s'agit du dernier manuscrit produit dans l'atelier de Christine, en 1418).

7 La Bibliographie générale (pp. 717-733) donne les références complètes de tous les ouvrages cités en abrégé dans l'inventaire; elle est suivie de nombreuses tables, qui permettent un repérage aisé de quelques informations importantes (pp. 735-768): incipits de $1^{\mathrm{er}}$ puis de $2^{\mathrm{e}} \mathrm{f}$; copistes; enlumineurs ornemanistes; peintres enlumineurs; table iconographique (où sont recensées des sujets récurrents: Christine de Pizan, scènes chrétiennes, courtoises, allégoriques, politiques et militaires, d'inspiration astrologique, mythologiques); table des possesseurs; index des noms de personnes; des noms d'artistes et artisans; manuscrits cités. Il est certainement trop sévère d'ajouter qu'une table des titres aurait sans doute rendu service. Un album final réunit une cinquantaine de planches en couleur.

8 Un inventaire de ce genre constituera certainement la base essentielle pour les recherches futures sur Christine de Pizan et sur l'histoire du livre manuscrit au début $\mathrm{du} \mathrm{XV}^{\mathrm{e}}$ siècle; si l'on peut se permettre un souhait, ce serait pour la réalisation d'une étape ultérieure, recensant les autres copies - ni autographes ni «originales» - des œuvres de Christine, ce qui permettrait de mesurer, d'une part, le caractère certes exceptionnel de sa propre production, et de l'autre les caractéristiques de copies non directement surveillées par l'auteur mais transmettant ses œuvres. 